\title{
Secure pathways for women in the UK: lessons from the women's enhanced medium secure services (WEMSS)
}

DOI:

10.1080/14789949.2016.1244279

\section{Document Version}

Accepted author manuscript

Link to publication record in Manchester Research Explorer

\section{Citation for published version (APA):}

Edge, D., Walker, T., Meacock, R., Wilson, H., McNair, L., Shaw, J., Gutridge, K., Mitchell, H., Robinson, L., Senior, J., Sutton, M., \& Abel, K. (2016). Secure pathways for women in the UK: lessons from the women's enhanced medium secure services (WEMSS). The Journal of Forensic Psychiatry \& Psychology, 1-20. https://doi.org/10.1080/14789949.2016.1244279

Published in:

The Journal of Forensic Psychiatry \& Psychology

\section{Citing this paper}

Please note that where the full-text provided on Manchester Research Explorer is the Author Accepted Manuscript or Proof version this may differ from the final Published version. If citing, it is advised that you check and use the publisher's definitive version.

\section{General rights}

Copyright and moral rights for the publications made accessible in the Research Explorer are retained by the authors and/or other copyright owners and it is a condition of accessing publications that users recognise and abide by the legal requirements associated with these rights.

\section{Takedown policy}

If you believe that this document breaches copyright please refer to the University of Manchester's Takedown Procedures [http://man.ac.uk/04Y6Bo] or contact uml.scholarlycommunications@manchester.ac.uk providing relevant details, so we can investigate your claim.

\section{OPEN ACCESS}




\section{DOI: $10.1080 / 14789949.2016 .1244279$}

Journal: The Journal of Forensic Psychiatry \& Psychology

Manuscript ID: 1244279

File Correction Details

Correction is made. No of Corrections: 28

Online Correction Link : http://tandfproofs.sps.co.in/oxe_tf_v3/index.php?

token=.MYBgq8TYAkmBanXI1JVGjDGSaFNT hxHaFeQ0jqvt NZbO6jkOxGRijA

\section{Image Annotation Details}

No Details Found

\section{Attached File Details}

No Details Found

\section{List of Comments}

\begin{tabular}{|c|c|c|}
\hline S.No & Text & Comments \\
\hline 1 & authorOrAffiliationGroup & Please change to 'Division of Psyhcology \& Mental Health' \\
\hline 2 & $\begin{array}{l}\text { three WEMSS pilots were opened } \\
\text { in } 2007 \text {, strategically placed } \\
\text { across the North, South and } \\
\text { Midlands }\end{array}$ & $\begin{array}{l}\text { Please replace current text with: } \\
\text { "...., three WEMSS pilots (strategically placed across the North, South } \\
\text { and Midlands) were opened in } 2007 \text { with a....." }\end{array}$ \\
\hline 3 & & insert 'care' \\
\hline 4 & . & $\begin{array}{l}\text { Please replace deleted text with: } \\
\text { Case finding criteria, derived from the original WEMSS population, } \\
\text { were used to select a control group of women with comparable } \\
\text { clinical and risk characteristics from three NHS medium secure } \\
\text { services, three independent medium secure services and one NHS } \\
\text { high secure service. }\end{array}$ \\
\hline 5 & & please insert 'levels of' \\
\hline 6 & minded & please replace with 'guarded' \\
\hline 7 & present & please replace with 'espousedd' \\
\hline 8 & , & please change comma for semi-colon \\
\hline 9 & , & please change comma to semi-colon \\
\hline
\end{tabular}




\begin{tabular}{|c|c|c|}
\hline 10 & . & please insert 'hopsitals' after Broadmoor \\
\hline 11 & $\begin{array}{l}\text { Lack of formal qualifications > } \\
\text { GCSEs }\end{array}$ & $\begin{array}{l}\text { The labels for these } 2 \text { variables have been transposed. This should } \\
\text { read 'Lack of successful long-term employment' }\end{array}$ \\
\hline 12 & $\begin{array}{l}\text { Lack of successful long-term } \\
\text { employment }\end{array}$ & $\begin{array}{l}\text { The lables for these } 2 \text { variables have been transposed. This should } \\
\text { read 'Lack of formal qualifications > GCSEs' }\end{array}$ \\
\hline 13 & $40(77 \%)$ & $\begin{array}{l}\text { Please amend data for this variable (Previous psychiatric inpatient } \\
\text { care }>1 \text { year) to read: } \\
42(80 \%) 62(78 \%) 1.000\end{array}$ \\
\hline
\end{tabular}

Query Details

1. Please check whether the author name 'Matthew Sutton' has been set correctly.

I confirm author name 'Matthew Sutton' has been set correctly

2. Would you consider changing the phrase "medium (high, low) secure" to "medium-( high-, low-)secure" in the article, when it behaves like an adjective? Please check, and correct if necessary.

I am happy to accept this recommendation. However, unable to make suggested changes in the text.

3. The usage of "time point(s)" and "timepoint(s)" seems to be inconsistent. Please check, and correct if necessary.

'timepoints' preferred - unable to edit text

4. Please provide the significance of the indicators (*) in Tables 2,3 , and 5.

I am unclear what is being requested. Significance values indicated in the table.

Statement about significance levle at the end of methods section as follows:

"Any references to statistically significant differences refer to the standard level of $p=.05$ unless otherwise stated".

5. The disclosure statement has been inserted. Please correct if this is inaccurate.

confirm no potential conflict of interest

6. The CrossRef database (www.crossref.org/) has been used to validate the references. Mismatches between the original manuscript and CrossRef are tracked in red font. Please provide a revision if the change is incorrect. Do not comment on correct changes.

Confirm references have been checked and amended/futher information provided where available

7. The reference 'Craissati and Taylor, 2014' is listed in the references list but is not cited in the text. Please either cite the reference or remove it from the references list and also provide missing city and page numbers for the same.

Please remove reference

8. Please provide missing city for the 'Jepson et al., 2001' references list entry. Missing city $=$ York

9. Please provide missing page numbers for the 'Pirkis et al. 2005 ' references list entry. Unable to find page numbers in original article or any previous citations 
doi:10.1186/1477-7525-3-76 


\section{Secure pathways for women in the UK: Iessons from the women's enhanced medium secure services (WEMSS) pilots}

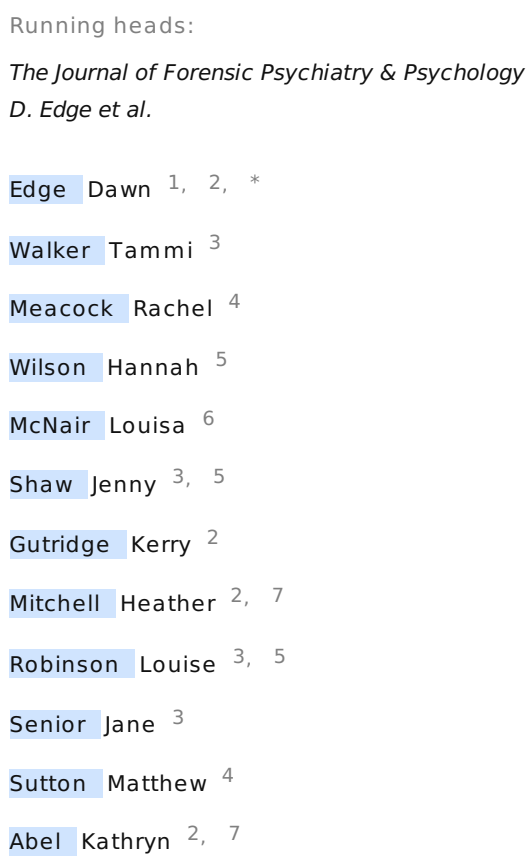

${ }^{3}$ Centre for Mental Health and Risk, Institute of Brain, Behaviour and Mental Health, University of Manchester, UK

${ }^{4}$ Manchester Centre for Health Economics, Institute of Population Health, University of Manchester, UK

5 Lancashire Care NHS Foundation Trust, Preston, UK

${ }^{6}$ Cheshire and Wirral Partnership NHS Foundation Trust, Chester, UK

${ }^{7}$ Manchester Mental Health \& Social Care Trust, UK

*Corresponding author. Email: dawn.edge@manchester.ac.uk

Received 26 Feb 2016; Accepted 25 Sep 2016

(c) 2016 Informa UK Limited, trading as Taylor \& Francis Group

\section{Abstract}

Women's enhanced medium secure services (WEMSS) is a model of care aimed at providing a more appropriate level of security for women and, in pilots became operational. This study compared the clinical outcomes of women in WEMSS with control women in six standard medium secure services and one high secure service matched on key clinical and risk characteristics, in order to examine their pathways of care. Our findings confirm that the WEMSS pilots were successful in transitioning women from high secure services who had previously been thought unsuitable for medium secure services. However, WEMSS showed no additional clinical benefit, suggesting that these women could be cared for equally well within standard medium secure services. We make recommendations about WEMSS and the future shape of women's secure care in England.

\section{Keywords}

Forensic mental health women's enhanced medium secure services (WEMSS) medium secure services high secure services gender-sensitive services care pathways

\section{Introduction}

In England, women's secure psychiatric care is delivered at three levels of security: high, medium and low. Until 2003, the 150 women detained in High Secure Hospitals (Ashworth, Broadmoor and Rampton) shared the sites with men and were subject to the same physical and procedural security. These arrangements came under scrutiny with growing disquiet about women's vulnerability in mixed-sex services and an emerging consensus that few women in high secure services posed 'grave and immediate danger', the main criterion for high secure detention. Service users', policy-makers', advocates' and staff concerns about women in high secure services were endorsed by a Special Hospitals Service Authority's report (Howlett, 1994 ) which confirmed that the majority of women did not warrant the highest level of security.

Almost a decade later, Shaw, Davies and Morey (2001) reported that little progress had been made with nearly $80 \%$ of women being held inappropriately in high secure services. The Department of Health acknowledged a lack of suitable alternatives for 'high risk' women and the inability of services, principally designed for men, to meet women's physical, psychological and emotional needs. The publication of seminal policy documents Women's Mental Health: Into the Mainstream (Department of Health, 2002) and a Women's Mental Health Strategy (Department of Health, 2004 ) promoted development of gender-appropriate psychiatric care and accommodation, as well as calling for a reduction in the number of women in high secure services and the development of services at lower levels of security. This increased the pressure to reduce the number of women's high secure beds in 
In response, three specialist women's enhanced medium secure services (WEMSS) were commissioned to provide care for an initial group of 46 women with complex mental health needs (encompassing complex comorbities, prolonged history of involvement with care/criminal justice/mental health systems and high risk of harm to the self or others) who could not be managed safely or effectively within standard medium secure services. These beds were commissioned to accommodate women discharged following the closure of women's services at Broadmoor and Ashworth. The evidence-base for these services was limited (Jepson, Di Blasi, Wright, \& Ter Reit, 2001 ) and although women's movement between different security levels was supposedly defined by risk and clinical need-; there was a lack of information about the measurement of these key determinants of security placements- or about how they influenced care pathways, themselves poorly defined. In the context of limited knowledge, three WEMSS pilots were opened in 2007, strategically placed across the North, South and Midlands Please replace current text with:

\section{"...., three WEMSS pilots (strategically placed across the North, South and Midlands) were opened in 2007 with}

a...." with a requirement for future independent evaluation (Edge, 2005).

Prior to implementation, stakeholders were consulted in a series of focus groups and individual interviews to discuss and identify relevant topics for the WEMSS evaluation. Stakeholders included women who had used; or were currently using services; carers of women in secure care; practitioners and service providers; policy-makers in mental health-and prison health and commissioners. All respondents agreed that therapeutic engagement would be enhanced in WEMSS, with a seven-day care delivery regime in which all activities would be 'therapeutic'.

An important component of WEMSS was that it should deliver relational, rather than physical security (Edge, 2005). Relational security involves staff translating their knowledge of the patient and the environment into high quality care and appropriate responses. Good relational security includes setting clear boundaries, engaging with patients regularly and committing to their treatment, having up-to-date care plans and understanding a patient's history, risk and triggers. WEMSS pilots also committed to enhancing physical environments, staffing, women's quality of life and reducing lengths of stay.

WEMSS was envisaged as a transparent process, facilitating women's pathways into and out of secure services. As stakeholders expressed concerns about the potential lack of a common approach resulting from independent development of WEMSS, the Department of Health commissioned an independent evaluation of WEMSS' clinical- and cost-effectiveness. This evaluation focused on the delivery of 'women-centred' outcomes to facilitate evidence-based assessment of future secure provision for women and to determine whether 'enhanced' services should become a standard component of care.

\section{Present study}

This study was embedded in the Department of Health-commissioned evaluation of the three WEMSS pilots. Key objectives were:

(1)

To describe the clinical and risk profiles of WEMSS women compared to a matched control of women in medium secure and high secure services;

(2)

To describe the differences in their care pathways.

Full care pathways are reported only for WEMSS women and women in comparator medium secure services, as the evaluation focused on this comparison.

\section{Methods}

Design

Mixed methods were used to evaluate the WEMSS pilots. Data collection took place between December 2010 and May 2011 . Here, we focus on the women's care pathways and their determinants, describing the referral sources, demographic details, risk and clinical characteristics, as well as the philosophies of care across WEMSS and control sites.

\section{Population}

The original pilots did not randomise women to WEMSS versus standard medium or high secure services. Therefore, to minimise bias, we used a casecontrol design to compare clinical outcomes and referral pathways of 'matched' women who had been in medium secure-or high secure insert 'Care' over the same period as those in WEMSS and, like the WEMSS women, had remained in the service for $\geq 12$ months. This time frame was considered the minimum amount of time required to demonstrate women's improvement within services.

In order to-select a control group of women with comparable elinieal and risk characteristics from three NHS medium secure services, three independent medium secure services and one NHS high secure service, case finding criteria were derived from the original wEMSS population. Please replace deleted text with:

Case finding criteria, derived from the original WEMSS population, were used to select a control group of women with comparable clinical and risk characteristics from three NHS medium secure services, three independent medium secure services and one NHS high secure service. These criteria were subdivided into primary and secondary criteria depending on their prevalence in WEMSS women (see Table 1 ), with more emphasis being placed on primary criteria for identifying comparable women.

Table 1. Primary and secondary case-finding criteria.

\begin{tabular}{|l}
\hline Primary criteria \\
Risk \\
- History of violent offending \\
- - Institutional violence \\
- $\cdot$ History of self-harm \\
- $\cdot$ HCR20 mean clinical scores within range of 5-8 (on admission) \\
- HCR20 mean risk scores within range of 6-9 (on admission) \\
\hline
\end{tabular}

Secondary criteria

Clinical

- Diagnosis of emotionally unstable personality disorder +/- diagnosis of major mental illn

- Multiple convictions

- History of arson

- History of weapon use 
- History of institutional care before the age of 1 8

Needs

- HoNOS scores within range of 11.5-22.4 (on admission)

- HoNOS Secure scores within range of 17-21 (on admission)

- History of disruption/truancy at school

- - Lack of formal qualifications beyond GCSES
- No children, or no access to their children

- Lack of successful em ployment (either no hist ory of employment or only short-term)

To reduce potential bias further, comparator women were selected from services based on location, with a preference for sites furthest away from WEMSS sites. This meant it would be more likely that the comparator women would have been selected for WEMSS, had the service been geographically available. Women from medium secure services provided within the same building/site as any WEMSS were excluded since co-location of facilities and personnel could increase the likelihood of contamination of the comparator/control through shared ethos/practice across WEMSS and non-WEMSS facilities. Screening of non-WEMSS women was completed by staff from the Mental Health Research Network (the NIHR research support network) and the research team using case note review and discussions with clinicians. Those classed as ' $m$ atches' then formally became the control participants in the study (comparator women)

\section{Dat a collection}

Standardised measures were used to collect data on risk and clinical outcomes. The Brief Psychiatric Rating Scale (BPRS), Beck Depression Inventory (BDI-II) and Camberwell Assessment of Need Forensic Version (CANFOR-S) were scored during the evaluation by two research assistants, using multidisciplinary case notes completed at the time of the woman's first Care Programme Approach (timepoint 1; TP1) and third Care Programme Approach (timepoint 2; TP2), approximately 12 months later. The case notes were daily records of care, which all members of staff contributed to (e.g. nurses, psychiatrists). A pro forma was used to extract information for each participant to ensure that similar information was obtained for all women. Both research assistants were psychology graduates with experience of women's medium secure settings. They received regular clinical supervision and discussed any queries with research team members to ensure accuracy and agreement on items. The Health of the Nation Outcome Scale (HoNOS) and HoNOS secure were part of routine data collected by clinicians and were included if they approximately corresponded to the required time

AQ3 $\mathbf{3}$ points please insert (TP) after 'timepoints'

For both groups of women, case notes were also used to obtain demographic details e.g. age, ethnicity, marital status, educational/employment histories, medication, clinical data on mental health (diagnoses, Mental Health Act status, duration and severity of illness, comorbidities), and details of their care pathways. Data were collected on the referral sources from which women had accessed their current service, duration of stay in current service and in other secure settings (including prison) and details of where women went on discharge.

Descriptions of the philosophies of care/models at each of the participating sites were obtained from documentation supplied by individual sites.

\section{St andardised measures}

The HoNOS was used to assess mental/social health needs (Wing et al., 1998). The HoNOS is rated out of 48 , with a higher score indicating more severe problems. The scale has moderately high internal consistency; Cronbach's $\alpha$ ranging from .59 to .76 (Pirkis et al., 2005 ).

The HoNOS Secure Version (Dickens, Sugarman, \& Walker, 2007) was used alongside the HoNOS to assess needs specific to secure environments. The HoNOS is rated out of 28 , with a higher score indicating more severe problems. The HoNOS-secure has been shown to have acceptable reliability (Dickens et al., 2007). HoNOS and HoNOS-secure had high rates of missing data, but were included as they provided a clinician-rated measure of clinical risk and need.

The Historical Clinical Risk Management 20 (HCR-20; Webster, Douglas, Eaves, \& Hart, 1997) was intended to be used to measure changes in risk. Unfortunately, the HCR-20 was not routinely collected by clinicians and therefore had very high please insert 'levels of' missing data.

The BPRS (Overall \& Gorham, 1962) was used to measure psychiatric symptoms e.g. anxiety, depression and hallucinations. It consists of 24 items, each of which is rated on a scale of $0-7$, where 0 indicates a symptom is not present and 7 indicates that a symptom is extremely severe. For the BPRS, items 15-24 were omitted because they are usually scored based on observations made during an interview (e.g. mannerisms/posturing) and this information was not available from case notes. BPRS scores reported in this paper are therefore out of 14 items rather than 24 .

The BDI-II (Beck, Steer, \& Brown, 1996 ) was used to measure depressive symptoms. The BDI-II consists of 21 symptoms, each of which is scored from 0 to 3 , with a higher score indicating increased symptom severity. The BDI-Il has high internal consistency and a retest reliability ranging from .73 to .96 (Wang \& Gorenstein, 2013 ).

The CANFOR-S (Thomas et al., 2003 ) was used to assess a range of health, social, clinical and functional needs. The full CANFOR-S consists of 25 items covering a broad range of needs, each of which is rated 'no problem', a 'met need', an 'unmet need', 'not applicable' or 'unknown'. For the CANFOR$\mathrm{S}$, items covering transport and accommodation were excluded because they were deemed irrelevant for the population. To maintain consistency, items with a missing data rate which exceeded $50 \%$ were excluded including basic education and benefits items, such that CANFOR-S scores rated out of a possible 21 instead of 25 needs.

\section{Analysis}

In the absence of randomisation, it would be misleading to compare average outcomes between the two groups at TP 2 as differences may simply reflect differences between the groups that were not covered by case-finding criteria. Therefore, we report the changes in outcomes over time.

SPSS Version 19 was used for the statistical analysis. Student's $t$-tests were used to examine statistical significance for continuous variables. The analysis of clinical and risk outcome measures involved multiple statistical comparisons. We have not performed a formal correction for multiple hypothesis testing and, given the small sample sizes, we are minded please replace with 'guarded' in our interpretation of statistical significance.

We have not attempted to impute missing data, but instead control statistically for bias caused by non-random missing data. When changes in scores over time were missing for more than $5 \%$ of individuals, we used a random effects model to estimate the difference in the mean scores between available observations for TP1 and for TP2. These results were compared to the test of the mean change in scores over time within individuals who were observed at 
both timepoints.

Any references to statistically significant differences refer to the standard level of $p=.05$ unless otherwise stated.

\section{Et hics}

Ethical approval was granted by County Durham \& Tess Valley NHS REC (10/H0905/13). All services also provided site-specific approval. Data collection was supported under section 251 of the NHS Act 2006 (ECC 6-05 (f)) to process patient identifiable information without consent. This was required because the small population size available meant it was essential that all participants' data were included.

\section{Results}

\section{St udy population}

All women admitted to WEMSS between June 2007 (WEMSS inception) and November $2009(n=59)$ were assessed. Of these women, 52 remained in WEMSS for $\geq 12$ months. During the study period, 214 women were admitted to comparator services. Seventy-nine were matched with WEMSS women on the case-finding criteria and had remained in the same facility for $\geq 12$ months.

\section{Demographics and referral sources}

Of the 52 women who had remained in WEMSS > 12 months, 30 were admitted from high secure; 10 were transferred from standard medium secure; eight from prison; three from low secure and one from another service.

When WEMSS first opened, a further 37 women had been considered for admission. Nineteen were declined because they were deemed 'unsuitable'; six could not be admitted through lack of funding; six were accepted but not admitted; four applications were withdrawn and two were being processed at the time of the study.

Of the 79 comparator women, 45 were in independent sector medium secure services, 23 were in NHS medium secure services and 11 were in NHS high secure. Table 2 shows the demographic characteristics and referral sources of WEMSS and comparator women.

Table 2. Demographics and referral source of women in WEMSS with a stay of longer than 12 months and comparator women.

\begin{tabular}{|c|c|c|c|}
\hline & WEMSS $(N=52)$ & Comparator $(N=79)$ & $p$ Value $($ Ho:WEMSS = WEMSS-like $)$ \\
\hline \multicolumn{4}{|l|}{ Age at TP1 } \\
\hline Mean (SD) & $36.35(8.77)$ & $27.92(7.91)$ & $0.000 *$ \\
\hline \multicolumn{4}{|l|}{ Ethnicity } \\
\hline White British & $41(79 \%)$ & $65(82 \%)$ & 0.135 \\
\hline Non-white British & $11(21 \%)$ & $8(10 \%)$ & \\
\hline \multicolumn{4}{|l|}{ Marital status } \\
\hline Single & $43(83 \%)$ & $63(80 \%)$ & 1.000 \\
\hline Other & $9(17 \%)$ & $14(18 \%)$ & \\
\hline \multicolumn{4}{|l|}{ Source of referral } \\
\hline LSS & $3(6 \%)$ & $8(10 \%)$ & $0.000 *$ \\
\hline MSS & $10(19 \%)$ & $19(24 \%)$ & \\
\hline HSS & $30(58 \%)$ & $6(8 \%)$ & \\
\hline HMP & $8(15 \%)$ & $32(41 \%)$ & \\
\hline Other & $1(2 \%)$ & $9(11 \%)$ & \\
\hline
\end{tabular}

Notes: LSS: low security services; MSS: medium secure services; HSS: high security services; HMP: Her Majesty's Prison. The following data was

AQ9 missing for women in the comparator group: age of $1 \%$, ethnicity of $8 \%$, marital status of $3 \%$ and source of referral of $6 \%$.

There were no significant differences between the two groups on ethnicity and marital status. However, WEMSS women were significantly older than comparator women.

\section{Risk characteristics}

Both groups had histories of violent offending, institutional violence, alcohol and/or substance abuse (see Table 3 ) at levels which did not differ significantly between groups. Although the percentage of women who self-harmed was high in both WEMSS ( $90 \%$ ) and the comparator services ( $100 \%$ ), it was significantly higher in the comparator group $(p=.005)$.

Table 3. Primary risk and diagnostic criteria of women in WEMSS with a stay of $>12$ months and comparator women.

\begin{tabular}{|c|c|c|c|}
\hline Variable & WEMSS $(n=52)$ & $\underset{(n=79)}{\text { Comparator }}$ & $\begin{array}{c}\text { p Value } \\
\text { (Ho:WEMSS } \\
=\text { WEMSS- } \\
\text { like) }\end{array}$ \\
\hline \multicolumn{4}{|l|}{ Risk } \\
\hline History of violent offending & $51(98 \%)$ & $76(96 \%)$ & 1.000 \\
\hline History of institutional violence & $47(90 \%)$ & $76(96 \%)$ & .432 \\
\hline History of self-harm & $47(90 \%)$ & $79(100 \%)$ & $.005^{*}$ \\
\hline
\end{tabular}




\begin{tabular}{|c|c|c|c|}
\hline $\begin{array}{l}\text { History of alcohol and/or substance abuse } \\
\text { Variable }\end{array}$ & WEMSS $45(n=52)$ & $\begin{array}{l}73(92 \%) \\
\text { Comparator }\end{array}$ & \\
\hline Clinical need & & & $\begin{array}{l}\text { (Hike) } \\
\text { = WEMSS- }\end{array}$ \\
\hline Diagnosis of personality disorder & $40(77 \%)$ & $65(82 \%)$ & .374 \\
\hline Diagnosis of emotionally unstable personality disorder & 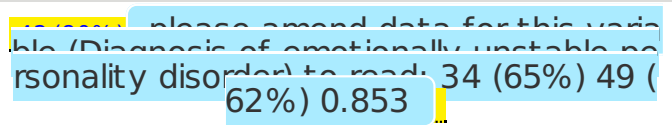 & $62(78 \%)$ & 1.000 \\
\hline Previous psychiatric inpatient care $>1$ year & 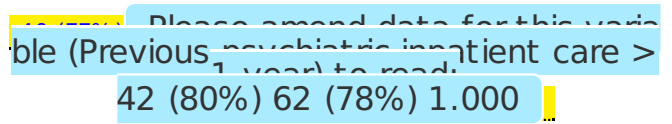 & $65(82 \%)$ & .374 \\
\hline History of childhood abuse & $\begin{array}{l}\text { variable (History of }- \text { hildhood abuse) to } r \\
46(88 \%) 75(95 \%) 0.155\end{array}$ & $62(78 \%)$ & 1.000 \\
\hline HoNOS mean (SD) & $15.86(6.74)$ & $16.92(5.96)$ & .496 \\
\hline HoNOS-secure mean (SD) & $19.65(3.74)$ & $19.02(3.62)$ & .417 \\
\hline History of school disruption and/or truancy & $34(65 \%)$ & $64(81 \%)$ & .563 \\
\hline $\begin{array}{l}\text { thring } \\
\text { in -hnitd mad 'Lack of successful long-term } \\
\text { employment' }\end{array}$ & $20(38 \%)$ & $62(78 \%)$ & $.035^{*}$ \\
\hline $\begin{array}{l}\text { Thir -hnnild read 'Lack of formal qualification' } \\
\mathrm{S}>\text { GCSES' }\end{array}$ & $39(75 \%)$ & $67(85 \%)$ & .331 \\
\hline \multicolumn{4}{|l|}{ Antipsychotic medication (DDD) } \\
\hline Mean DDD TP1 (SD) & $2.65(2.72)$ & $1.59(1.97)$ & $.011 *$ \\
\hline Mean DDD TP2 (SD) & $1.74(2.50)$ & $1.04(1.69)$ & .058 \\
\hline Mean within individual change (TP2-TP1) (SD) & $-.91(3.37)$ & $-.58(1.98)$ & .484 \\
\hline$p$ Value $(\mathrm{HO}: \Delta=0)$ & .057 & $.011 *$ & \\
\hline
\end{tabular}

Notes: DDD: Levels of antipsychotic medication expressed in Defined Daily Dose. The following data were missing for WEMSS women: history of institutional violence of $2 \%$, previous psychiatric inpatient care $>1$ year of $4 \%$, HoNOS of $57.69 \%$, HoNOS-Secure of $28.85 \%$, history of school disruption and/or truancy of $23 \%$, lack of formal qualifications > GCSE for $13 \%$ and lack of successful long-term employment for $48 \%$. The following data were missing for comparator women: diagnosis of PD of $1 \%$, diagnosis of emotionally unstable PD of $1 \%$, previous psychiatric inpatient care $>1$ year of $15 \%$, history of childhood abuse of $1 \%$, HoNOS of $24.05 \%$, HoNOS secure of $27.85 \%$, history of school disruption and/or truancy of $6 \%$, lack of formal qualifications > GCSE for $9 \%$ and lack of successful long-term employment for $15 \%$.

WEMSS and comparator women also had similar levels of clinical need: there were no significant differences between the groups on diagnosis of personality disorder, previous psychiatric inpatient care $>1$ year, history of childhood abuse, mean HoNOS and HoNOS-Secure scores, history of school disruption and/or truancy or lack of successful long-term employment. The proportion of women with formal qualification > GCSE was significantly higher in the comparator group (78\%) than WEMSS $(38 \%)(p=.035)$. Please amend text as follows:

'Although similar in terms of educational attainment, the proportion of women lacking successful long-term employment was significantly higher in the comparator group $(78 \%)$ than WEMSS $(38 \%)(p=.035)^{\prime}$

At TP1 levels of antipsychotic medication (mean defined daily dose) were higher in WEMSS $(M=2.65)$ than comparator women $(M=1.59)(p=.011)$. Only the comparator group had a significant reduction in medication levels from TP1 $(M=1.59)$ to TP2 $(M=1.04)(p=.011)$.

\section{Philosophies of care}

All sites provided documentation evidencing their philosophies/models of care (see Table 4 ). All sites explicitly mentioned gender-sensitive practice, recovery and the importance of multi-disciplinary team work. However, only two sites (both WEMSS) mentioned following a seven-day-a-week model.

\begin{tabular}{|c|c|c|c|c|c|c|c|c|c|c|}
\hline & \multicolumn{3}{|c|}{ WEMSS } & \multicolumn{3}{|c|}{ NHS HSS } & \multicolumn{3}{|c|}{ Independent sector MSS } & \multirow{2}{*}{$\begin{array}{c}\text { HSS } \\
\text { Site J }\end{array}$} \\
\hline & Site A & Site B & Site C & Site D & Site E & Site F & Site G & Site H & Site I & \\
\hline \multicolumn{11}{|l|}{ Models of care } \\
\hline Psycho-social interventions & $x$ & & $x$ & & & & & $\mathrm{x}$ & & \\
\hline DBT & $x$ & & $x$ & $\mathrm{x}$ & & & $\mathrm{x}$ & $\mathrm{x}$ & $\mathrm{x}$ & \\
\hline Attachment theory & $x$ & $\mathrm{X}$ & $x$ & & $x$ & $x$ & $x$ & & & $x$ \\
\hline Risk assessment & $x$ & & & & & & & $\mathrm{x}$ & & \\
\hline Trauma models & & & & & & $x$ & & $\mathrm{X}$ & & \\
\hline Mentalisation-based treatment (MBT) & & $x$ & & & & & & & & \\
\hline Psvchodvnamic MDT function & $\mathrm{x}$ & $\mathrm{x}$ & $x$ & & & & & & & \\
\hline
\end{tabular}




\begin{tabular}{|c|c|c|c|c|c|c|c|c|c|c|}
\hline Integrated need-adapted approach & \multirow[b]{2}{*}{ Site A } & \multirow{2}{*}{$\begin{array}{c}\underset{X}{\text { WEMSS }} \\
\text { Site B }\end{array}$} & \multirow[b]{2}{*}{$\underset{X}{\text { Site C }}$} & \multirow[b]{2}{*}{ Site D } & NHS HSS & \multirow[b]{2}{*}{ Site F } & \multicolumn{3}{|c|}{ Independent sector MSS } & \multirow{2}{*}{$\begin{array}{l}\text { HSS } \\
\text { Site }\end{array}$} \\
\hline Diathesis-stress model & & & & & Site E & & Site G & Site $\mathbf{H}$ & Site I & \\
\hline Recovery model & $\mathrm{x}$ & & $\mathrm{x}$ & $x$ & & & & $x$ & & \\
\hline Social learning theory & $\mathrm{x}$ & & & & & & $\mathrm{x}$ & & $\mathrm{x}$ & \\
\hline \multicolumn{11}{|l|}{ Key elements of model stated in documentation } \\
\hline 7-days/week approach & $\mathrm{x}$ & & $\mathrm{x}$ & & & & & & & \\
\hline Whole environment / system approach & $x$ & & $\mathrm{x}$ & $\mathrm{x}$ & & & & & & \\
\hline CPA & $x$ & $\mathrm{x}$ & $x$ & $\mathrm{x}$ & $x$ & & $x$ & $\mathrm{X}$ & $x$ & $\mathrm{x}$ \\
\hline Meaningful day/activity & $x$ & & $x$ & & $x$ & & $x$ & $\mathrm{x}$ & & $x$ \\
\hline Gender sensitive care & $x$ & $\mathrm{x}$ & $x$ & $x$ & $x$ & $\mathrm{X}$ & $x$ & $x$ & $x$ & $x$ \\
\hline Patient-centred/empowerment & $x$ & $x$ & $\mathrm{x}$ & $x$ & & $x$ & $x$ & $x$ & $x$ & $x$ \\
\hline Recovery & $x$ & $x$ & $x$ & $x$ & $x$ & $x$ & $x$ & $\mathrm{x}$ & $x$ & $x$ \\
\hline Psycho-social integration & & $x$ & & & & $x$ & & & $x$ & $x$ \\
\hline Relational security & $x$ & $x$ & $x$ & $x$ & $x$ & $x$ & & $x$ & & $x$ \\
\hline Enhanced physical health care & $x$ & $x$ & $\mathrm{x}$ & & & & & & & \\
\hline Risk assessment & $x$ & $\mathrm{x}$ & $\mathrm{X}$ & & $x$ & $x$ & $x$ & $\mathrm{x}$ & & $x$ \\
\hline Staffing/MDT important & $\mathrm{x}$ & $\mathrm{x}$ & $x$ & $\mathrm{x}$ & $\mathrm{x}$ & $\mathrm{x}$ & $\mathrm{x}$ & $x$ & $\mathrm{x}$ & $\mathrm{x}$ \\
\hline Family/carer involvement & $x$ & $\mathrm{x}$ & $\mathrm{x}$ & $\mathrm{x}$ & $\mathrm{x}$ & $x$ & $\mathrm{x}$ & $\mathrm{x}$ & & $\mathrm{x}$ \\
\hline Purpose-built facilities & $x$ & $x$ & $x$ & & & & $x$ & $\mathrm{x}$ & $x$ & $x$ \\
\hline Outcome measures stated & & $x$ & & $x$ & & & & & & \\
\hline KPI stated & & & & $x$ & $x$ & & $x$ & & & \\
\hline Admission/exclusion criteria & $x$ & & & $x$ & $x$ & & $x$ & $x$ & & $x$ \\
\hline Discharge criteria/planning & $x$ & & & $\mathrm{x}$ & $\mathrm{x}$ & & & $\mathrm{x}$ & & $\mathrm{x}$ \\
\hline
\end{tabular}

Notes: DBT: Dialectical Behavioural Therapy; MDT: Multidisciplinary team; CPA: Care Programme Approach; KPI: Key Performance Indicator.

The majority of sites included relational security as a key element of their model. Six of the sites highlighted the importance of having admission and discharge criteria, but this was only clearly articulated in 1 non-WEMSS service.

\section{Clinical out comes}

Table 5 reports clinical outcomes. Mean total BDI-II and BPRS scores were significantly higher in the comparator group than WEMSS at both timepoints. The WEMSS group had a significant reduction in BDI-II scores over time, but the change over time was not significant for the comparator group. Conversely, the reduction in BPRS scores over time was only significant in the comparator group.

Table 5. Clinical outcomes of WEMSS and comparator women.

\begin{tabular}{|c|c|c|c|}
\hline & $\begin{array}{l}\text { WEMSS } \\
(n=52)\end{array}$ & $\underset{(n=79)}{\text { Comparator }}$ & $p$ Value $($ Ho:WEMSS $=$ WEMSS - \\
\hline$B D I-/ /$ total score & Mean (SD) & Mean (SD) & \\
\hline TP1 & $12.43(8.27)$ & $18.22(7.58)$ & $<.001 *$ \\
\hline TP2 & $9.43(7.38)$ & $16.57(7.61)$ & $<.001^{*}$ \\
\hline Within individual change TP2-TP1 & $-3.57(9.64)$ & $-1.65(7.89)$ & .230 \\
\hline$p$ Value $(\mathrm{HO} 0: \Delta=0)$ & $.016^{*}$ & .068 & \\
\hline $\begin{array}{l}\text { Random effects estimate of the mean change in available observations } \\
\text { (TP2-TP1) }\end{array}$ & $-3.01(1.59)$ & & \\
\hline (se) & $(1.59)$ & & \\
\hline$p$ Value $(\mathrm{H} 0: \Delta=0)$ & .062 & & \\
\hline \multicolumn{4}{|l|}{ BPRS total score Q.s1-14 } \\
\hline TP1 & $34.90(8.32)$ & $39.66(7.78)$ & $.001 *$ \\
\hline TP2 & $32.48(9.72)$ & $36.99(9.26)$ & $.009 *$ \\
\hline Within individual change TP2-TP1 & $-2.27(10.63)$ & $-2.67(9.01)$ & .820 \\
\hline$p$ Value $(\mathrm{HO} 0: \Delta=0)$ & .133 & $.010 *$ & \\
\hline CAMIEOD_C & & & \\
\hline
\end{tabular}




\begin{tabular}{|c|c|c|c|}
\hline \multicolumn{4}{|l|}{ ב-איוט ועוחר } \\
\hline No. of met needs TP1 & $\begin{array}{r}\text { WEMSS } \\
7.15 \eta 2.452)\end{array}$ & $\begin{array}{l}\text { Comparator } \\
7.15(1.4 \xi 79)\end{array}$ & $\begin{array}{l}\text { p Value }(\text { Ho: WEMSS = WEMSS } \\
.996\end{array}$ \\
\hline No. of unmet needs TP1 & $4.42(1.81)$ & $5.10(1.89)$ & $.043 *$ \\
\hline No. of total needs TP1 & $11.58(2.00)$ & $12.25(2.12)$ & .070 \\
\hline No. of met needs TP2 & $7.63(2.11)$ & $7.92(2.34)$ & .460 \\
\hline No. of unmet needs TP2 & $3.63(2.37)$ & $4.04(2.11)$ & .311 \\
\hline No. of total needs TP2 & $11.27(2.30)$ & $11.96(1.95)$ & .067 \\
\hline Within individual change in met needs TP2-TP1 & $.48(2.56)$ & $.77(2.23)$ & .491 \\
\hline$p$ Value $(\mathrm{H} 0: \Delta=0)$ & .181 & $.003 *$ & \\
\hline Mean within individual change in unmet needs TP2-TP1 (SD) & $-.79(2.48)$ & $-1.06(2.40)$ & .528 \\
\hline$p$ Value $(\mathrm{H} 0: \Delta=0)$ & $.026 *$ & $.000 *$ & \\
\hline Mean within individual change in total needs TP2-TP1 (SD) & $-.31(2.27)$ & $-.29(1.69)$ & .962 \\
\hline$p$ Value $(\mathrm{H} 0: \Delta=0)$ & .333 & .129 & \\
\hline \multicolumn{4}{|l|}{ HONOS } \\
\hline Mean total score TP1 (SD) & $15.86(6.74)$ & $16.92(5.96)$ & .496 \\
\hline Mean total score TP2 (SD) & $12.98(6.30)$ & $14.86(4.71)$ & .076 \\
\hline Mean within individual change TP2-TP1 (SD) & $-3.30(6.11)$ & $-2.11(6.34)$ & .468 \\
\hline$p$ Value $(\mathrm{H} 0: \Delta=0)$ & $.026 *$ & $.016 *$ & \\
\hline $\begin{array}{l}\text { Random effects estimate of the mean change in available observations } \\
\text { (TP2-TP1) }\end{array}$ & -2.86 & -2.06 & .447 \\
\hline (se) & $(1.67)$ & $(.96)$ & \\
\hline$p$ Value $(\mathrm{H} 0: \Delta=0)$ & .089 & $.034 *$ & \\
\hline \multicolumn{4}{|l|}{ HoNOS Secure } \\
\hline Mean total score TP1 (SD) & $19.65(3.74)$ & $19.02(3.62)$ & .417 \\
\hline Mean total score TP2 (SD) & $17.41(3.80)$ & $17.64(3.71)$ & .763 \\
\hline Mean within individual change TP2-TP1 (SD) & $-2.55(4.27)$ & $-1.53(3.36)$ & .229 \\
\hline$p$ Value $(\mathrm{H} 0: \Delta=0)$ & $.002 *$ & $.002 *$ & \\
\hline $\begin{array}{l}\text { Random effects estimate of the mean change in available observations } \\
\text { (TP2-TP1) }\end{array}$ & -2.24 & -1.37 & .242 \\
\hline (se) & $(.87)$ & $(.68)$ & \\
\hline$p$ Value $(\mathrm{H} 0: \Delta=0)$ & $.012 *$ & $.046 *$ & \\
\hline
\end{tabular}

Notes: The following data were missing for women in the WEMSS group; BDI-II scores of $2 \%$ at TP1, BDI-II Scores of $10 \%$ at TP 2 , BDI-II change scores of $12 \%$, BPRS scores of $2 \%$ at TP1, BPRS change scores of $2 \%$, HoNOS scores of $58 \%$ at TP 1 , HoNOS scores of $12 \%$ at TP 2 , HoNOS change scores of $62 \%$, HoNOS-secure scores of $29 \%$ at TP1, HoNOS-secure scores of $25 \%$ at TP2 and HoNOS-secure change scores of $37 \%$. The following data were missing for women in the comparator group; HoNOS scores of $24 \%$ at TP 1 , HoNOS scores of $19 \%$ at TP2, HoNOS change scores of $29 \%$, HoNOS-secure scores of $28 \%$ at TP1, HoNOS-secure scores of $26 \%$ at TP2, HoNOS-secure change scores of $35 \%$..

There was no significant difference in the mean number of met and total needs (CANFoR-S) between WEMSS and comparator women at TP1 or in the changes over time for both groups. However, the mean number of unmet needs was significantly higher for the comparator group than WEMSS at TP 1 but not TP2. There was a significant reduction in the mean number of unmet needs between TP1 and TP2 for both groups. The comparator group also showed a significant increase in the number of met needs between TP1 and TP2.

There were high levels of missing data on HCR-20, HoNOS and HoNOS-secure. The HCR-20 was missing $8 \%$ of WEMSS SCores at TP $1,41 \%$ of WEMSS scores at TP2, $59 \%$ of comparator scores at TP 1 and $81 \%$ of comparator scores at TP 2 . The HoNOS was missing $58 \%$ of WEMSS Scores at TP 1 , $12 \%$ of WEMSS scores at TP2, $24 \%$ of comparator scores at TP 1 and $19 \%$ of comparator scores at TP 2 . The HoNOS-secure was missing $29 \%$ of WEMSS scores at TP1, 25\% of WEMSS scores at TP2, $28 \%$ of comparator scores at TP1 and $26 \%$ of comparator scores at TP 2.

Total HoNOS scores did not differ significantly between groups at either time point. There was a significant reduction in mean total HoNOS scores between TP1 and TP2 for both groups, but no significant difference in these changes between the two groups. HoNOS Secure scores also fell significantly between TP1 and TP2 in WEMSS and comparator women, but again, there was no significant difference in these changes between the two groups. Missing HoNOS data provided only $38 \%$ of the WEMSS and $71 \%$ of the comparator group with observations at both time points. A random effects model was used to examine the extent of any bias introduced into our estimates by missing data. Results did not differ significantly from those of individuals only observed once, suggesting that there is no systematic bias.

\section{Care pathways following discharge}

WEMSS women had greater mean (757 vs. 645 days) and median (719 vs. 680) lengths of stay than comparator women (Table 6 ). Of the few women discharged during the study period, a similar proportion of WEMSS and comparator women had a 'positive' transfer to lower level of security, including transfer from WEMSS to medium secure (three WEMSS (6\%) and 11 comparator women (16\%) transferred to low secure, five WEMSS women ( $8 \%$ ) 
transferred to medium secure). Two women in the comparator group (4\%) transferred to alternative medium secure services. Three women from WEMSS $(6 \%)$ moved to higher security, compared with no comparator women.

Table 6. Discharge locations of women in WEMSS and comparator women from MSS services.

\begin{tabular}{|c|c|c|}
\hline & WEMSS $(n=52)$ at $1 / 10 / 10$ & Comparator MSS $(n=68)$ at $1 / 10 / 10$ \\
\hline Women discharged/left service (\% of total sample) & $12(23 \%)$ & $14(21 \%)$ \\
\hline Mean Length of stay of women discharged/left service (days) & 756.75 & 645.43 \\
\hline Median Length of stay of women discharged/left service (days) & 719 & 605 \\
\hline Range of women discharged/left service (days) & $381-1071$ & $440-979$ \\
\hline \multicolumn{3}{|l|}{ Location of discharge } \\
\hline Community & - & - \\
\hline LSS & $3(6 \%)$ & $11(16 \%)$ \\
\hline MSS & $5(8 \%)$ & $2(3 \%)$ \\
\hline WEMSS & - & - \\
\hline Prison & - & - \\
\hline HSS & $3(6 \%)$ & - \\
\hline Died & $1(2 \%)$ & $1(1 \%)$ \\
\hline
\end{tabular}

The full pathways (admission to discharge) of the women in WEMSS and the medium secure comparator women ( $n=68$ ) are depicted in Figure 1 .

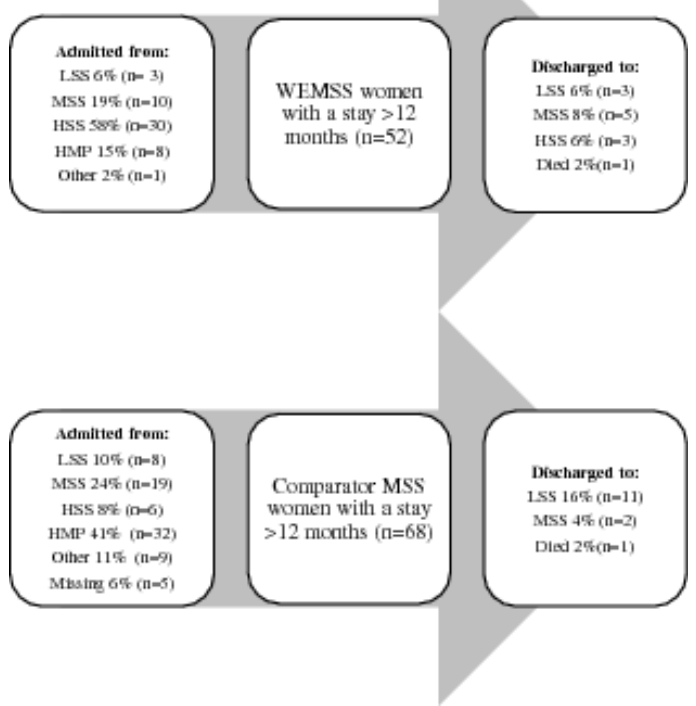

\section{Discussion}

This evaluation of the WEMSS pilots assessed the care pathways of WEMSS versus comparator women with similar clinical and risk characteristics.

The main findings are:

(1)

Clinical outcomes of both groups were similar. The significant differences between the two groups were: higher BDI-II and BPRS scores at both time points in the comparator group; a reduction in BDI-II scores over time only in the WEMSS group; a reduction in BPRS scores over time only in the comparator group; higher unmet needs at TP1 in the comparatorgroup and an increase in the number of met needs over time only in the comparator group.

(2)

Women in WEMSS typically stayed longer than women in comparator services (757 vs. 645 days).

(3)

A similar proportion of both groups transferred to lower security levels. However, three WEMSS women transferred to higher security versus no comparator women.

(4)

Philosophies/models of care varied across sites. All sites highlighted relational security, multidicrinlinan toammenrk nender-cencitive practice and recovery as key components of their model. A 7-day-a-week approach was present please replace with 'espousedd' in two WEMSS services. 
Our findings suggest that WEMSS successfully fulfilled its initial remit to manage the transition of women from high secure services. Given that the pilots were developed to manage this transition, it is not surprising that 3 years after WEMSS' inception, high secure remained the predominant source of referral (58\%). The importance of this should not be underestimated. Women previously held at the highest level of security, either as a result of their risk profile- or because they had been considered 'too difficult to manage' in standard medium secure services, were able to transfer to this lower security level, please change comma for semi-colon albeit enhanced. In the sample of WEMSS women, three were discharged to low secure and five to medium secure.. please change comma to semi-colon transfers which may not have been possible had they remained in high secure.

Movement of women from high secure services to WEMSS facilitated closure of women's beds at Ashworth and Broadmoor.: please insert 'hopsitals' after Broadmoor Arguably, this has been the catalyst for a new framework in which women with complex needs and staff can talk meaningfully about, and plan for, discharge to lower security levels. We note that $42 \%$ of women in WEMSS had been admitted from elsewhere in the secure forensic system. This supports perceptions that there were (are) significant numbers of 'WEMSS-like' women housed elsewhere across the secure women's estate.

Although meeting their aim to decrease the size of the women's high secure estate, our data suggest the WEMSS pilots did not meet other success criteria. Prior to inception, stakeholders envisaged that the services would enhance women's recovery pathways, improving their mental health and wellbeing (Edge, 2005). We found little difference in clinical outcomes between WEMSS and comparator women. Furthermore, we found little evidence that WEMSS provided women with enhanced relational security and more intensive therapeutic activities. As a result of the large amount of missing clinical data, further research is required to confirm or refute our findings that WEMSS does not provide greater clinical benefit to these vulnerable, complex women.

Additionally, stakeholder expectation of faster movement through the secure care pathway and of women returning to the community more quickly via WEMSS was not met. There was little evidence of more movement from WEMSS into lower security care compared to standard medium secure. Overall, WEMSS women remained in their service for longer than comparator women. Three WEMSS women transferred to higher security, compared with none in comparator services. This may be a reflection of the WEMSS' women's history within forensic services, and perceptions of their 'riskiness'. Alternatively, the lack of movement to lower security levels may reflect a lack of suitable accommodation in terms of bed numbers or geographical location (Bartlett, Walker, Harty, \& Abel, 2014 ). Such concerns were raised at the time by clinicians and continue to be raised by staff working in low or medium secure settings (Somers \& Bartlett, 2014). Another possibility is that WEMSS services had fewer and less established links with lower security services. Longitudinal studies and process mapping are required to understand fully why so few WEMSS women were transferred to lower security.

Our evaluation raises important questions regarding pathways for women into and out of WEMSS. In the 3 years since inception, 39 women were declined admission to WEMSS with 19 deemed 'unsuitable'. However, only one of the three WEMSS services articulated the importance of clear admission criteria in their philosophy of care. Women and commissioners require clear admission criteria, together with signposting for potential pathways through services. This would improve understanding of the degree to which 'WEMSS women' are distinct from women requiring standard medium secure and clarify the clinical or other characteristics which determine whether women should be managed in enhanced settings.

The WEMSS pilots were developed independently of each other and adopted different models of care and service delivery. The data collected in this study on models of care across all WEMSS and medium secure services highlight the variation in WEMSS services. Consequently, it was difficult to draw meaningful comparisons between the possible clinical benefits of treatment programmes in WEMSS and comparator services.

\section{St rengt hs}

This is the first study to formally compare care pathways and clinical outcomes of women in enhanced medium secure services with matched women in standard services. The case-control design enabled us to minimise bias resulting from a lack of randomisation and to take account of many key variables which might have explained differences between services. This detailed evaluation has allowed a far better contemporary understanding of the path of complex women through forensic services as well as providing insight into the need for better information to plan future provision.

\section{Limit ations}

An important limitation was the fact that women were not randomised into the pilots. We are aware that such research methodologies are challenging within clinical services. We addressed the lack of randomisation by matching the comparator group to the WEMSS group using evidence-based, casefinding criteria, derived from the characteristics of the original WEMSS group. In addition, we sought comparator women from locations where they would not have access to WEMSS. We also conducted an analysis focusing on women's improvements in outcomes over time. In the tendering process for this study, there was a limited time frame for the evaluation. This meant that we were only able to provide a limited follow-up period and are aware that a longer time frame to assess clinical benefit may be particularly appropriate for this group of women.

A further limitation was the sample size ( 52 WEMSS vs. 79 comparator). This was, of course, limited by the duration and extent of the commissioned pilots. Our ability to detect differences between the two groups and the possibility of Type II errors should be borne in mind. To mitigate this imposed sampling problem, all available participants were included in the analysis. However, our evaluation was also limited by significant missing data on some measures e.g. HoNOS. Poor data collection was not confined to standard medium secure; rather it was also found in WEMSS. This was unexpected given the additional service costs and their explicit pilot status.

Data were collected from case notes with researchers relying on the available information to complete some outcome measures (BPRS, BDI-II, CANFOR-S) retrospectively. We would encourage future clinical pilots to be performed through randomised controlled trials with the opportunity for standardised, prospective data collection on predetermined measures.

\section{Future provision}

The 2012 Care Act resulted in major reconfiguration of the NHS from 2013 when NHS England assumed responsibility for commissioning all secure inpatient care for adults in England. This included the three existing WEMSS services, which continue to provide 36 secure care beds for women nationally. NHS England recognises that the WEMSS pilots evaluation was conducted at a point in time and that approaches to care delivery and commissioning models have developed significantly as the understanding of particular patient cohorts and associated management approaches has matured (personal communication). In 2015, NHS England reviewed the strategic approach for women's secure services and concluded that an updated national commissioning strategy was required to underpin delivery of an integrated women's secure pathway model spanning high, medium and low secure care. Work by NHS England Specialised Commissioning is currently underway to deliver a pathway that embodies the core principles of gender-specific care in least restrictive settings, as close to home as possible and informed by the WEMSS evaluation. 
This evaluation suggests that 'WEMSS-like' women can be cared for in standard medium secure services without undermining clinical and risk outcomes. Contrary to our expectation, women in standard medium secure services experience broadly similar outcomes as women in WEMSS. None of the women in comparator services had been referred from high secure. This may imply that staff in medium secure services are reluctant to admit women perceived to be too dangerous or too complex for medium secure; or that staff in high secure services are reluctant to refer to medium secure. This evaluation concludes that many of the features of enhanced care could be, and likely are being, delivered in standard medium secure. This suggests that with the right support, medium secure teams could also receive women from high secure services.

We were unable to identify detailed evidence of how decisions were made about the admission of women to WEMSS. Whilst most study sites highlighted the importance of clear admission and discharge criteria, these were only clearly outlined in one comparator medium secure service. We would recommend that future services have well-defined admission and discharge criteria to aid decision-making and facilitate movement through the care pathway.

This evaluation also found that routine collection of the pre-specified bundle of standardised outcome measures was lacking, in pilot WEMSS as well as comparator services. If WEMSS continue to be a part of the specialist commissioning, we recommend a clear framework with shared, transparent admission and discharge criteria, specific benchmarked evaluation criteria, as well as standard clinical pathways and outcomes akin to the care programme approach used in acute mental health services. Such a process would facilitate delivery of meaningful, high-quality data to specialist commissioners and policy-makers.

\section{Conclusion}

This independent, case-controlled evaluation found little difference between care pathways outcomes for WEMSS and comparator women. This included no evidence of additional clinical benefit. Given the lack of differences between the groups in their movement through secure care, if WEMSS remains part of women's secure provision, its position within the pathway should be clearly specified. This evaluation highlights the importance of systematic collection of valid and reliable outcome measures with which to facilitate future evidence-based service development. A decade on from inception of WEMSS pilots, further research is required to determine the extent to which their goal has been achieved and whether such services represent value for money.

\section{Disclosure statement}

AQ4 No potential conflict of interest was reported by the authors.

\section{Funding}

This work was supported by the Department of Health, UK.

\section{Acknowledgments}

The study was commissioned by and received financial support from the Department of Health, UK. However, the views expressed are those of the authors and do not necessarily reflect those of the funders.

\section{Ref erences}

Bartlett , A. , Walker , T. , Harty , M. A. , \& Abel , K. M. (2014). Health and social care services for women offenders: Current provision and a future model of AQ5 care. The Journal of Forensic Psychiatry \& Psychology, 25, 625-635.

Beck, A. T. , Steer , R. A. , \& Brown , G. K. (1996). Manual for the BDI-II. San Antonio, TX: Psychological Corporation.

Craissati , J. , \& Taylor , P. (2014). Forensic mental health services in the United Kingdom and Ireland. In J. Gunn \& P. Taylor (Eds.), Forensic psychiatry: Clinical, AQ6 legal and ethical issues. CRC Press.

Department of Health. (2002). Women's mental health: Into the mainstream - Strategic development of mental health care for women. London: Author.

Department of Health (2004). Women's mental health strategy. London: Author.

Dickens , G. , Sugarman , P. , \& Walker , L. (2007). HoNOS-secure: A reliable outcome measure for users of secure and forensic mental health services. Journal of Forensic Psychiatry \& Psychology, 18, 507-514. Edge, D. (2005). Women's enhanced medium secure services (WEMSS): A scoping exercise for developing a research and evaluation strategy. London: Departm ent of

Howlett, M. (1994). Special hospitals service authority service for women patients. London: SHSA.

Jepson , R. , Di Blasi , Z. , Wright, K. , \& Ter Reit , G. (2001). Scoping of the effectiveness of mental health services (CRD Report 21). NHS Centre for Review and AQ7 University of York, Dissemination.

Overall , J. E. , \& Gorham , D. R. (1962). The Brief Psychiatric Rating Scale. Psychological Reports, 10, 799-812.

Pirkis, J. E. , Burgess , P. M. , Kirk, P. K. , Dodson, S. , Coombs , T. J. , \& Williamson , M. K. (2005). A review of the psychometric properties of the Health of the A08 8 Nation Outcome Scales (HoNOS) family of measures. Health and Quality of Life Outcomes, 76.

Reed , J. (1994). Report of the working group on high security and related psychiatric provision. London: Department of Health.

Shaw , J. , Davies , J. , \& Morey , H. (2001). An assessment of the security, dependency and treatment needs of all patients in secure services in a UK health region. The Journal of Forensic Psychiatry, 12, 610-637.

Somers, N. , \& Bartlett, A. (2014). Women's secure hospital care pathways in practice: A qualitative analysis of clinicians views in England and Wales. BMC Health Services Research, 14, 590-600. 
Thomas , S. , Harty , M. A. , Parrott , J. , McCrone , P. , Slade , M. , \& Thornicroft , G. (2003). Camberwell assessment of need-Forensic version. London: Gaskell.

Wang , Y. P. , \& Gorenstein , C. (2013). Psychometric properties of the Beck Depression Inventory-II: A comprehensive review. Revista Brasileira de Psiquiatria, 35, $416-43 i$.

Webster , C. D. , Douglas , K. S. , Eaves , D. , \& Hart , S. D. (1997). HCR-20. Assessing the risk of violence. Version 2. Burnaby: Sim on Fraser University and Forensic Psychiatric Services Comm ission of British Columbia.

Wing , J. K. , Beevor , A. S. , Curtis , R. H. , Park , S. B. G. , Hadden , S. , \& Burns , A. (1998). Health of the Nation Outcome Scales (HoNOS): Research and developm ent. The British Journal of Psychiatry, 172, 11-18. 